MATHEMATICS OF COMPUTATION

Volume 72, Number 242, Pages 525-539

S $0025-5718(02) 01426-6$

Article electronically published on March 21, 2002

\title{
MIXED FINITE VOLUME METHODS ON NONSTAGGERED QUADRILATERAL GRIDS FOR ELLIPTIC PROBLEMS
}

\author{
SO-HSIANG CHOU, DO Y. KWAK, AND KWANG Y. KIM
}

\begin{abstract}
We construct and analyze a mixed finite volume method on quadrilateral grids for elliptic problems written as a system of two first order PDEs in the state variable (e.g., pressure) and its flux (e.g., Darcy velocity). An important point is that no staggered grids or covolumes are used to stabilize the system. Only a single primary grid system is adopted, and the degrees of freedom are imposed on the interfaces. The approximate flux is sought in the lowest-order Raviart-Thomas space and the pressure field in the rotated- $Q 1$ nonconforming space. Furthermore, we demonstrate that the present finite volume method can be interpreted as a rotated- $Q 1$ nonconforming finite element method for the pressure with a simple local recovery of flux. Numerical results are presented for a variety of problems which confirm the usefulness and effectiveness of the method.
\end{abstract}

\section{INTRODUCTION}

Let $\Omega$ be a bounded polygonal domain in $\mathbb{R}^{2}$ with the boundary $\partial \Omega$. We consider the second-order elliptic boundary value problem

$$
\left\{\begin{aligned}
-\operatorname{div}(\mathcal{K} \nabla p)=f & & \text { in } \Omega, \\
p=0 & & \text { on } \partial \Omega,
\end{aligned}\right.
$$

where $\mathcal{K}=\mathcal{K}(\mathbf{x})$ is a symmetric and uniformly positive definite matrix, i.e., there exist two positive constants $c_{1}$ and $c_{2}$ such that

$$
c_{1} \xi^{T} \xi \leq \xi^{T} \mathcal{K}(\mathbf{x}) \xi \leq c_{2} \xi^{T} \xi, \quad \forall \xi \in \mathbb{R}^{2}, \mathbf{x} \in \bar{\Omega} .
$$

Let us introduce the vector variable $\mathbf{u}=-\mathcal{K} \nabla p$ and rewrite the problem (1.1) in the mixed form

$$
\left\{\begin{array}{rlrl}
\mathbf{u}+\mathcal{K} \nabla p & =0, & & \text { in } \Omega, \\
\operatorname{div} \mathbf{u}=f, & & \text { in } \Omega, \\
p=0, & & \text { on } \partial \Omega .
\end{array}\right.
$$

In the mathematical modeling of fluid flow in porous media, $\mathbf{u}$ and $p$ represent the velocity and pressure fields, respectively. The first equation of (1.2), which

Received by the editor November 14, 2000 and, in revised form, May 29, 2001.

2000 Mathematics Subject Classification. Primary 65F15, 65N30, 35J60.

Key words and phrases. Mixed method, finite volume method, box method, quadrilateral grid.

The research of the first author was supported by NSF grant DMS-0074259.

The research of the second and third authors was supported by BK21 project, Korea and by grant No. 2000-2-10300-001-5 from the Basic Research Program of the Korea Science \& Engineering Foundation. 
relates $\mathbf{u}$ and $p$, is called Darcy's law, and the second equation represents the conservation of mass. In the full system of equations for certain porous media problems such as transport of contaminants or tracers, these equations are coupled with the concentration equation. Since the coupling is only through the velocity variable $\mathbf{u}$, it is important to gain very accurate approximation for the velocity $\mathbf{u}$ when discretizing the full system of equations. More details about this point can be found, for example, in [18, 30].

Much effort has been made in accurate computation of the velocity variable since the late 1970's. The mixed finite element method, which is the standard finite element method for the system (1.2), has been a very active area of research, and many finite element spaces subject to the well-known inf-sup stability condition have been developed; see, for example, 3 4, 5, 19, 20, 24, 28, 33. On the other hand, the finite volume method has been also applied to the system (1.2) in several ways. For example, see [6, 11, 12, 25] for mixed covolume methods, and [35, 36, 38] for different approaches.

One shortcoming of some of the early mixed methods mentioned above is that they led to indefinite matrix systems when discretizing (1.2). This prevents one from applying well known iterative methods such as the conjugate-gradient method that are useful to solve the symmetric positive definite matrix system. This drawback causes severe difficulties particularly in solving the porous media problem, because one has to solve the mixed system (1.2) at every time step to obtain the approximate velocity which appears in the concentration equation (cf. [18, 30]).

A common way to remedy this situation is to make use of numerical quadratures for calculating integrals (which is adopted in [35, 36] 38]). This enables one to decouple the pressure $p$ easily and obtain a symmetric positive definite matrix system for $p$ only which is very similar to cell-centered finite difference methods. Another way is to introduce the Lagrange multipliers on the edges of the mesh to ensure the continuity of normal components of the velocity variable [22]. In this fashion the velocity and the pressure finite element spaces have no continuity constraints at all, and thus both variables can be eliminated to obtain a symmetric positive definite matrix system which only involves the Lagrange multipliers. It can be shown that this matrix system is equivalent to some nonconforming finite element method for the original problem (1.1). For the interested readers we refer to [1] 7] 27.

On the other hand, Courbet and Croisille [17, seeking to avoid the inf-sup condition, considered the lowest-order Raviart-Thomas space for the velocity and the $P 1$ nonconforming element for the pressure, and discretized the mixed system (1.2) in the case of $\mathcal{K}=I$, the identity matrix, by the finite volume (box) method, using only a single triangular grid system. It was shown that a symmetric positive definite system in the unknown $p_{h}$ can be obtained with the flux recovered by a simple formula. It was later extended and analyzed in a more effective way by Chou and Tang [15] to general tensor-coefficient problems. Furthermore, following the previous successful and consistent viewpoint [11, 12, 16] that any given finite volume method should be related to a close finite element method, they provided in [14, 15], among other things, a new framework showing equivalence between mixed finite volume methods and nonconforming Galerkin methods with a cheap local recovery of the fluxes. The significance of this is that, unlike lower order mixed finite element methods, mixed finite volume methods can decouple the pressure 
from the flux and compute it basically cost free. However, all these results pertain to the triangular grid case, where one has the convenient area coordinates to use.

In the mixed finite volume literature, it is common to introduce a secondary grid or dual grid of covolumes to enforce equality between the numbers of equations and unknowns [6, 11, 12, 13, 38]. Thus it is rather surprising that there exist finite volume methods such as those in [14, 15, 17, achieving stability using a single grid system while violating the inf-sup condition on the pressure-velocity spaces. Let us now list some features of the mixed finite volume scheme on nonstaggered triangular grids:

- The discretized system gives rise to a mixed system in which the pressure decouples easily and the velocity can be recovered by a simple local formula.

- An accurate approximation for the velocity can be computed from the nonconforming approximation of the pressure in a direct manner, with no help from Lagrange multipliers. (This is a restatement of the first item from a different angle.)

- The nonconforming Galerkin method for the pressure requires fewer degrees of freedom than the mixed finite element method.

- The pressure solution can be obtained by a fast solver such as the multigrid algorithm (cf. 2, 8, 9, 26]).

- The data structure is simple, since only one mesh system is used.

- The mixed finite volume scheme satisfies discrete mass conservation locally, as all the mixed methods mentioned above do.

In view of the fact that many practical hydrocodes (e.g., [31, 32]) have been written using conservation laws over irregular polygonal control volumes such as triangles and quadrilaterals, it is useful to ask if one can construct and analyze the corresponding finite volume method on rectangular and/or quadrilateral grids that shares the above features. The answer turns out to be positive. The first step involves a correct choice of pressure and velocity spaces: we will make use of the lowest-order Raviart-Thomas space for the velocity variable [34, 39] and the rotated- $Q 1$ nonconforming element for the pressure [33. The second step involves an effective way of decoupling the mixed discrete system: we show that the velocity approximation $\mathbf{u}_{h}$ can be easily eliminated to yield the rotated- $Q 1$ nonconforming method for the pressure $p_{h}$ only, and that $\mathbf{u}_{h}$ can be recovered from $p_{h}$ in a simple manner.

It is sometimes believed that the use of finite volume methods is mainly in hyperbolic conservation laws and there is no need to use them for elliptic problems. For this reason, we mention that in an important book 29] McCormick has elucidated, among other things, the reasons why methods of the finite volume element type are natural and effective on composite grids in conjunction with the multilevel FAC iterative solver. In particular, they play useful roles in approximating elliptic problems.

The rest of the paper is organized as follows. In the next section we introduce some notations about the grids and define the relevant function spaces. In Section 3, we describe the mixed finite volume method on quadrilateral and rectangular grids, and we establish optimal error estimates for it in Section 4. Finally, in Section 5, some numerical results are presented to illustrate the efficiency of our method. 


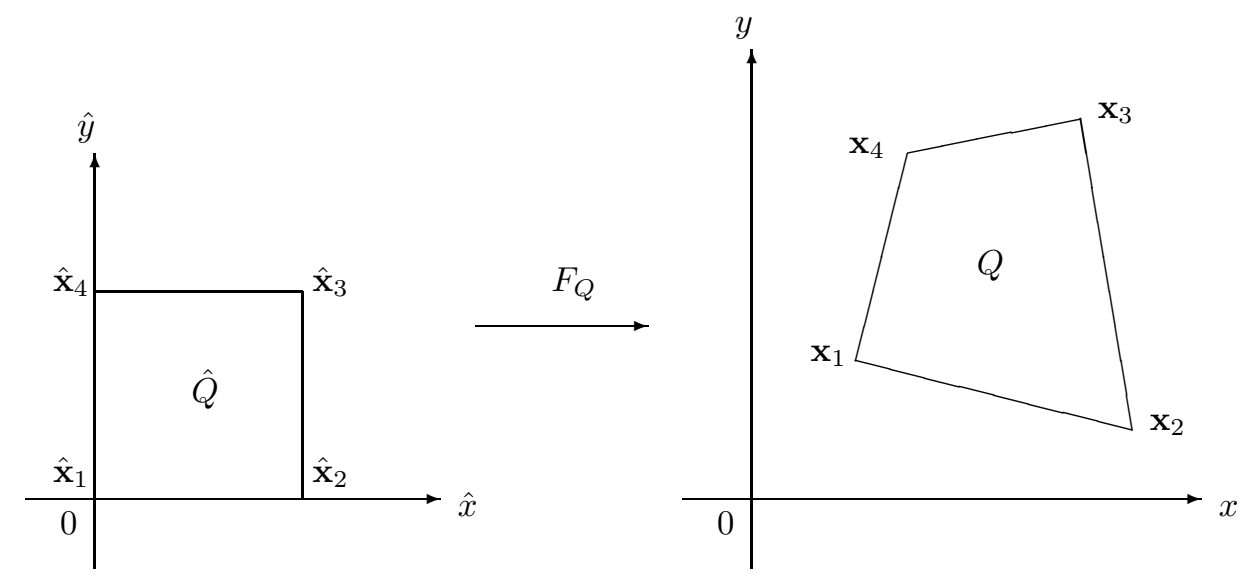

Figure 2.1. The bilinear mapping $F_{Q}: \hat{Q} \rightarrow Q$

\section{Preliminaries}

Let $\mathcal{Q}_{h}$ be a partition of $\Omega$ into convex quadrilaterals whose diameters are less than or equal to $h$. The intersection, if any, of any two (closed) quadrilaterals in the partition is either a common edge or a common vertex. Let

$$
\begin{aligned}
& N Q=\text { Number of elements of } \mathcal{Q}_{h}, \\
& N E_{i}=\text { Number of interior edges of } \mathcal{Q}_{h}, \\
& N E_{b}=\text { Number of boundary edges of } \mathcal{Q}_{h},
\end{aligned}
$$

and $N E=N E_{i}+N E_{b}$, the total number of edges of $\mathcal{Q}_{h}$.

Let $\hat{\mathbf{x}}=(\hat{x}, \hat{y})$ and $\mathbf{x}=(x, y)$. We use the unit square $\hat{Q}=[0,1] \times[0,1]$ as the reference element (cf. Figure 2.1) in the $\hat{x} \hat{y}$-plane with the vertices

$$
\hat{\mathbf{x}}_{1}=(0,0), \quad \hat{\mathbf{x}}_{2}=(1,0), \quad \hat{\mathbf{x}}_{3}=(1,1), \quad \hat{\mathbf{x}}_{4}=(0,1) .
$$

Let $Q$ be a convex quadrilateral with the vertices $\mathbf{x}_{i}$ arranged counterclockwise. Then there exists a unique invertible bilinear transformation $F_{Q}$ which maps $\hat{Q}$ onto $Q$ and satisfies

$$
\mathbf{x}_{i}=F_{Q}\left(\hat{\mathbf{x}}_{i}\right), \quad i=1,2,3,4
$$

In fact, it is given by

$$
\mathbf{x}=F_{Q}(\hat{\mathbf{x}})=\mathbf{x}_{1}+\mathbf{x}_{21} \hat{x}+\mathbf{x}_{41} \hat{y}+\mathbf{g} \hat{x} \hat{y}
$$

where we set

$$
\mathbf{x}_{i j}=\mathbf{x}_{i}-\mathbf{x}_{j}, \quad \mathbf{g}=\mathbf{x}_{12}+\mathbf{x}_{34} .
$$

The Jacobian matrix $\mathcal{J}_{Q}$ of $F_{Q}$ is given by

$$
\mathcal{J}_{Q}=\left(\begin{array}{ll}
\frac{\partial x}{\partial \hat{x}} & \frac{\partial x}{\partial \hat{y}} \\
\frac{\partial y}{\partial \hat{x}} & \frac{\partial y}{\partial \hat{y}}
\end{array}\right)=\left(\mathbf{x}_{21}+\mathbf{g} \hat{y}, \mathbf{x}_{41}+\mathbf{g} \hat{x}\right) .
$$

Denote by $S_{i}$ the subtriangle of $Q$ with vertices $\mathbf{x}_{i-1}, \mathbf{x}_{i}$ and $\mathbf{x}_{i+1}\left(\mathbf{x}_{0}=\mathbf{x}_{4}\right)$. Let $h_{Q}$ be the diameter of $Q$ and $\rho_{Q}=2 \min _{1 \leq i \leq 4}$ \{diameter of circle inscribed in $S_{i}$. 
Throughout the paper we assume a regular family of partitions $\mathcal{Q}=\left\{\mathcal{Q}_{h}\right\}$, i.e., there exists a positive constant $\sigma$ independent of $h$ such that

$$
\frac{h_{Q}}{\rho_{Q}} \leq \sigma, \quad \forall Q \in \mathcal{Q}_{h}, \forall \mathcal{Q}_{h} \in \mathcal{Q} .
$$

The following upper bounds for $\mathcal{J}_{Q}$ and $J_{Q}$ can be found, e.g., in [23]:

$$
\begin{aligned}
\left\|\mathcal{J}_{Q}\right\|_{\infty, \hat{Q}} \leq C h_{Q}, & \left\|\mathcal{J}_{Q}^{-1}\right\|_{\infty, Q} \leq C h_{Q}^{-1}, \\
\left|J_{Q}\right|_{\infty, \hat{Q}} \leq C h_{Q}^{2}, & \left|J_{Q}^{-1}\right|_{\infty, Q} \leq C h_{Q}^{-2},
\end{aligned}
$$

where $\|M\|_{\infty, K}:=\sup _{\mathbf{x} \in K}\|M(x)\|$, the supremum of the spectral norm of the matrix function $M$. Hereafter $C$ will denote a generic positive constant which is independent of $h$. It may have different values in different places, especially when used in proofs.

Simple calculation shows that the determinant $J_{Q}=\operatorname{det} \mathcal{J}_{Q}$ is a linear function of $\hat{x}$ and $\hat{y}$ :

$$
J_{Q}(\hat{x}, \hat{y})=\alpha+\beta \hat{x}+\gamma \hat{y}
$$

where

$$
\alpha=\operatorname{det}\left(\mathbf{x}_{21}, \mathbf{x}_{41}\right), \quad \beta=\operatorname{det}\left(\mathbf{x}_{21}, \mathbf{g}\right), \quad \gamma=\operatorname{det}\left(\mathbf{g}, \mathbf{x}_{41}\right) .
$$

We shall assume throughout the paper that each quadrilateral in the family of partitions is almost a parallelogram: $\|\mathbf{g}\|=O\left(h_{Q}^{2}\right)$. In other words,

The distance between the midpoints of the two diagonals of $Q$ is $O\left(h_{Q}^{2}\right)$.

This condition is easily satisfied if the partitions are obtained by symmetric refinement of quadrilaterals via bisection on edges.

It is well known that (shape) regularity is equivalent to the minimum angle condition for partitions with triangular elements. For the quadrilateral case, it is shown in 10 that the shape regularity condition (2.3) is equivalent to the following two conditions: the uniform boundedness of $h_{Q} / h_{Q}^{\prime}$, the ratios of diameter to shortest edge $h_{Q}^{\prime}$, and the existence of a positive constant $s$ independent of $h$ such that $\left|\cos \left(\theta_{Q}\right)\right| \leq s<1$ for all $Q$ with $\theta_{Q}$ any interior angle of $Q$. A similar result can be found in [37, which states that a certain quasi-regularity condition plus the almost parallelogram condition (2.8) imply the above two conditions. A detailed clarification on the relations between various regularity and uniformity conditions on quadrilateral grids can be found in 10 .

The Piola transformation $\mathcal{P}_{Q}$ transforms a vector-valued function on $\hat{Q}$ to one on $Q$ by

$$
\mathbf{v}=\mathcal{P}_{Q} \hat{\mathbf{v}}=\frac{1}{J} \mathcal{J} \hat{\mathbf{v}} \circ F^{-1}
$$

where we drop the subscript $Q$ for brevity. This transformation preserves the $H$ (div) space on the reference element and has the following well known properties (cf. [34, 39]): If we let $\hat{p}=p \circ F$, then

$$
\begin{aligned}
\int_{Q} \nabla p \cdot \mathbf{v} d x d y & =\int_{\hat{Q}} \hat{\nabla} \hat{p} \cdot \hat{\mathbf{v}} d \hat{x} d \hat{y}, \\
\operatorname{div} \mathbf{v} & =\frac{1}{J} \operatorname{div} \hat{\mathbf{v}} .
\end{aligned}
$$

We need the following lemma for error analysis. 
Lemma 2.1. Let $\mathbf{v}$ and $\hat{\mathbf{v}}$ be related by (2.9). For regular partitions, there exist positive constants $C_{1}$ and $C_{2}$ such that for every $\mathbf{v} \in \mathbf{L}^{2}(Q)$ we have

$$
C_{1}\|\mathbf{v}\|_{0, Q} \leq\|\hat{\mathbf{v}}\|_{0, \hat{Q}} \leq C_{2}\|\mathbf{v}\|_{0, Q} .
$$

If the regular partition satisfies the almost a parallelogram condition (2.8), then for every $\mathbf{v} \in \mathbf{H}^{1}(Q)$,

$$
|\mathbf{v}|_{1, Q} \leq C_{1} h^{-1}\|\hat{\mathbf{v}}\|_{1, \hat{Q}}, \quad|\hat{\mathbf{v}}|_{1, \hat{Q}} \leq C_{2} h\|\mathbf{v}\|_{1, Q} .
$$

Proof. The proof can be easily done by using the formulae (2.2), (2.7) and the bounds (2.4), (2.5).

Now we introduce the function spaces

$$
\begin{gathered}
\mathbf{V}=\mathbf{H}(\operatorname{div}, \Omega)=\left\{\mathbf{v} \in \mathbf{L}^{2}(\Omega): \operatorname{div} \mathbf{v} \in L^{2}(\Omega)\right\}, \\
\mathbf{H}^{s}(\operatorname{div}, \Omega)=\left\{\mathbf{v} \in \mathbf{L}^{2}(\Omega): \operatorname{div} \mathbf{v} \in H^{s}(\Omega)\right\} .
\end{gathered}
$$

To define the lowest-order Raviart-Thomas space $\mathbf{V}_{h}$ on $\mathcal{Q}_{h}$, let $\mathbf{V}_{h}(\hat{Q})$ denote the local space on the reference element $\hat{Q}$ :

$$
\mathbf{V}_{h}(\hat{Q})=\{\hat{\mathbf{v}}: \hat{\mathbf{v}}=(a+b \hat{x}, c+d \hat{y}), a, b, c, d \in \mathbb{R}\} .
$$

Then the local space $\mathbf{V}_{h}(Q)$ on each quadrilateral $Q$ is defined to be

$$
\mathbf{V}_{h}(Q)=\left\{\mathbf{v}=\mathcal{P}_{Q} \hat{\mathbf{v}}: \hat{\mathbf{v}} \in \mathbf{V}_{h}(\hat{Q})\right\},
$$

and the global space $\mathbf{V}_{h}$ is defined by

$$
\mathbf{V}_{h}=\left\{\mathbf{v} \in \mathbf{V}:\left.\mathbf{v}\right|_{Q} \in \mathbf{V}_{h}(Q), \forall Q \in \mathcal{Q}_{h}\right\} .
$$

Furthermore, the discrete space satisfies the condition that if $\mathbf{n}_{i}$ denotes the unit outward normal to the edge $e_{i}$ of $Q$, then

$$
\left|e_{i}\right| \mathbf{v} \cdot \mathbf{n}_{i}=\hat{\mathbf{v}} \cdot \hat{\mathbf{n}}_{i}, \quad i=1,2,3,4,
$$

where $\hat{\mathbf{n}}_{i}$ is the unit exterior normal to $\hat{e}_{i}$. Note that every $\mathbf{v} \in \mathbf{V}_{h}$ has continuous normal components across the edges of $\mathcal{Q}_{h}$, which are constant due to (2.18). We refer to 34 39] for further details.

The Raviart-Thomas projection $\Pi_{h}: \mathbf{V} \rightarrow \mathbf{V}_{h}$ is defined as follows: Let us define $\hat{\Pi}$ on $\hat{Q}$ to be

$$
\int_{\hat{e}} \hat{\Pi} \hat{\mathbf{v}} \cdot \hat{\mathbf{n}} d s=\int_{\hat{e}} \hat{\mathbf{v}} \cdot \hat{\mathbf{n}} d s, \quad \forall \text { edges } \hat{e} \text { of } \hat{Q},
$$

and then set

$$
\Pi_{Q} \mathbf{v}=\mathcal{P}_{Q}(\hat{\Pi} \hat{\mathbf{v}}), \quad \forall \mathbf{v} \in \mathbf{H}^{1}(Q),
$$

where $\mathcal{P}_{Q} \hat{\mathbf{v}}=\mathbf{v}$. Finally, we define

$$
\left.\Pi_{h} \mathbf{v}\right|_{Q}=\Pi_{Q} \mathbf{v} .
$$

Some well known properties [34 39] of $\Pi_{h}$, necessary to derive error estimates, are summarized in the following lemma.

Lemma 2.2. If $w \in L^{2}(\Omega)$ is a piecewise constant function on $\mathcal{Q}_{h}$, then

$$
\left(\operatorname{div}\left(\mathbf{u}-\Pi_{h} \mathbf{u}\right), w\right)=0, \quad \forall \mathbf{u} \in \mathbf{V} .
$$


Also, the following estimates are valid:

$$
\begin{gathered}
\left\|\mathbf{u}-\Pi_{h} \mathbf{u}\right\|_{0} \leq C h\|\mathbf{u}\|_{1}, \quad \forall \mathbf{u} \in \mathbf{H}^{1}(\Omega), \\
\left\|\operatorname{div}\left(\mathbf{u}-\Pi_{h} \mathbf{u}\right)\right\|_{0} \leq C h\|\operatorname{div} \mathbf{u}\|_{1}, \quad \forall \mathbf{u} \in \mathbf{H}^{1}(\operatorname{div} ; \Omega) .
\end{gathered}
$$

The pressure space $N_{h}$ is chosen to be the rotated- $Q 1$ nonconforming finite element space given by ([33])

$$
\begin{aligned}
N_{h}=\left\{p:\left.p\right|_{Q} \in N_{h}(Q), \forall Q \in \mathcal{Q}_{h} ; \text { and if } Q_{1}, Q_{2} \text { share an edge } e,\right. \\
\text { then } \left.\left.\int_{e} p\right|_{\partial Q_{1}} d s=\left.\int_{e} p\right|_{\partial Q_{2}} d s ; \text { and }\left.\int_{\partial Q \cap \partial \Omega} p\right|_{\partial \Omega} d s=0\right\},
\end{aligned}
$$

where the local spaces are defined by

$$
\begin{gathered}
N_{h}(\hat{Q})=\operatorname{span}\left\{1, \hat{x}, \hat{y}, \hat{x}^{2}-\hat{y}^{2}\right\}, \\
N_{h}(Q)=\left\{p=\hat{p} \circ F_{Q}^{-1}: \hat{p} \in N_{h}(\hat{Q})\right\} .
\end{gathered}
$$

The degrees of freedom for $N_{h}$ are given by $\left\{\frac{1}{|e|} \int_{e} p_{h} d s: e\right.$ is an edge of $\left.\mathcal{Q}_{h} \cdot\right\}$.

Lemma 2.3. We have

$$
\nabla N_{h}(\hat{Q})=\left\{\hat{\mathbf{v}} \in \mathbf{V}_{h}(\hat{Q}): \operatorname{div} \hat{\mathbf{v}}=0\right\} .
$$

Proof. The proof is obvious, since we have $\nabla N_{h}(\hat{Q})=\{(a+c \hat{x}, b-c \hat{y})\}$.

Finally, we define $\bar{f}_{h}$ to be the function given by $\left.\bar{f}_{h}\right|_{Q}=\frac{1}{J_{Q}} \int_{Q} f$. Note that only when $Q$ is a rectangle is $\left.\bar{f}_{h}\right|_{Q}$ the local average of $f$ on $Q$.

Lemma 2.4. Assume that $f \in H^{1}(Q), \forall Q \in \mathcal{Q}_{h}$. Then

$$
\left\|f-\bar{f}_{h}\right\|_{0} \leq C h\left(\sum_{Q}\|f\|_{1, Q}^{2}\right)^{1 / 2} .
$$

Proof. We first note that

$$
\left\|f-\bar{f}_{h}\right\|_{0, Q} \leq C h\left\|\hat{f}-\frac{1}{J_{Q}} \int_{\hat{Q}} J_{Q} \hat{f} d \hat{x}^{\prime} d \hat{y}^{\prime}\right\|_{0, \hat{Q}} .
$$

Writing $J_{Q}$ in the integrand as

$$
J_{Q}\left(\hat{x}^{\prime}, \hat{y}^{\prime}\right)=J_{Q}(\hat{x}, \hat{y})+\beta\left(\hat{x}^{\prime}-\hat{x}\right)+\gamma\left(\hat{y}^{\prime}-\hat{y}\right),
$$

we have

$$
\begin{aligned}
\hat{f}-\frac{1}{J_{Q}} \int_{\hat{Q}} J_{Q} \hat{f} d \hat{x}^{\prime} d \hat{y}^{\prime} & =\left[\hat{f}(\hat{x}, \hat{y})-\int_{\hat{Q}} \hat{f}\left(\hat{x}^{\prime}, \hat{y}^{\prime}\right) d \hat{x}^{\prime} d \hat{y}^{\prime}\right] \\
& +\int_{\hat{Q}} \frac{\beta\left(\hat{x}-\hat{x}^{\prime}\right)+\gamma\left(\hat{y}-\hat{y}^{\prime}\right)}{J_{Q}(\hat{x}, \hat{y})} \hat{f}\left(\hat{x}^{\prime}, \hat{y}^{\prime}\right) d \hat{x}^{\prime} d \hat{y}^{\prime} \\
& \equiv I_{1}+I_{2}
\end{aligned}
$$

Clearly, $I_{1}$ is bounded by $C|\hat{f}|_{1, \hat{Q}}$. Now by (2.7) one has $|\beta|+|\gamma|=O\left(h_{Q}^{3}\right)$, and the bound for $I_{2}$ follows easily. Combining these results gives

$$
\left\|f-\bar{f}_{h}\right\|_{0, Q} \leq C h\left(|\hat{f}|_{1, \hat{Q}}+h\|\hat{f}\|_{0, \hat{Q}}\right) \leq C h\|f\|_{1, Q} .
$$

Summing over $Q$ completes the proof. 


\section{Mixed finite Volume methods ON RECTANGULAR AND QUADRILATERAL GRIDS}

In this section we first introduce a finite volume method (FVM) on rectangular grids and relate it to a nonconforming finite element method with local recovery of the flux. The ideas are then generalized to quadrilateral grids.

3.1. FVM on rectangular grids. To define the finite volume method on rectangular grids, we begin by integrating the mixed system (1.2) over each element $Q \in \mathcal{Q}_{h}$ :

$$
\int_{Q}\left(\mathbf{u}_{h}+\mathcal{K} \nabla p_{h}\right)=0, \quad \int_{Q} \operatorname{div} \mathbf{u}_{h}=\int_{Q} f .
$$

This gives rise to a total of $3 N Q$ equations in $2 N E_{i}+N E_{b}$ unknowns. It is easy to see that this is a square matrix system on triangular grids (cf. [17]), since we have

$$
3 N Q=\sum_{Q} \sum_{\partial Q} 1=2 \sum_{e \in E_{i}} 1+\sum_{e \in E_{b}} 1=2 N E_{i}+N E_{b}
$$

However, on rectangular and quadrilateral grids we have $4 N Q=2 N E_{i}+N E_{b}$, which implies that $N Q$ additional equations, or equivalently, one additional equation per element, is required.

To resolve this problem we propose the following scheme: Find $\left(\mathbf{u}_{h}, p_{h}\right) \in \mathbf{V}_{h} \times$ $N_{h}$ which satisfies, on every element $Q \in \mathcal{Q}_{h}$,

$$
\left\{\begin{array}{c}
\int_{Q}\left(\mathbf{u}_{h}+\mathcal{K} \nabla p_{h}\right) \cdot \nabla \chi=0, \quad \forall \chi \in N_{h}(Q), \\
\int_{Q} \operatorname{div} \mathbf{u}_{h}=\int_{Q} f .
\end{array}\right.
$$

Note that this gives the desired number of equations, since we have $\operatorname{dim} \nabla N_{h}(Q)=$ 3. We also see that any solution $\left(\mathbf{u}_{h}, p_{h}\right)$ of the system (3.2) satisfies (3.1), since $\nabla N_{h}(Q)$ contains the constant vectors $(1,0),(0,1)$.

Now let us show how the velocity $\mathbf{u}_{h}$ is eliminated and the system (3.2) reduces to the nonconforming finite element method for $p_{h}$ only. Noticing that $\mathbf{u}_{h} \cdot \mathbf{n}$ is constant on the edges and $\chi \in N_{h}$ has common averages on the interior edges with vanishing boundary averages, we obtain, for every $\chi \in N_{h}$,

$$
\sum_{Q \in \mathcal{Q}_{h}} \int_{Q} \mathbf{u}_{h} \cdot \nabla \chi=\sum_{Q \in \mathcal{Q}_{h}}\left[\int_{\partial Q}\left(\mathbf{u}_{h} \cdot \mathbf{n}\right) \chi-\int_{Q} \operatorname{div} \mathbf{u}_{h} \chi\right]=-\int_{\Omega} \bar{f}_{h} \chi
$$

where we used the equality $\operatorname{div} \mathbf{u}_{h}=\bar{f}_{h}$. By virtue of (3.2), it follows immediately that

$$
\sum_{Q \in \mathcal{Q}_{h}} \int_{Q} \mathcal{K} \nabla p_{h} \cdot \nabla \chi=\int_{\Omega} \bar{f}_{h} \chi, \quad \forall \chi \in N_{h}
$$

This is a rotated- $Q 1$ nonconforming finite element method for the problem (1.1), except that the right-hand side is given by $\bar{f}_{h}$ instead of $f$.

The velocity $\mathbf{u}_{h}$ can be computed directly from the solution $p_{h}$ of (3.4) in the following manner. Let $Q$ be an arbitrary element of $\mathcal{Q}_{h}$ with the edges $e_{i}$, 
$i=1,2,3,4$, and let $\phi_{i} \in N_{h}(Q)$ be the basis function associated with the edge $e_{i}$, namely, $\frac{1}{\left|e_{i}\right|} \int_{e_{i}} \phi_{j}=\delta_{i j}$. Then the outflux through the edge $e_{i}$ is given by

$$
\begin{aligned}
\left.\left|e_{i}\right|\left(\mathbf{u}_{h} \cdot \mathbf{n}\right)\right|_{e_{i}} & =\int_{\partial Q}\left(\mathbf{u}_{h} \cdot \mathbf{n}\right) \phi_{i}=\int_{Q} \operatorname{div}\left(\mathbf{u}_{h} \phi_{i}\right) \\
& =\int_{Q}\left(\operatorname{div} \mathbf{u}_{h} \phi_{i}+\mathbf{u}_{h} \cdot \nabla \phi_{i}\right),
\end{aligned}
$$

from which it follows by the fact $\operatorname{div} \mathbf{u}_{h}=\bar{f}_{h}$ and (3.2) that

$$
\left.\left|e_{i}\right|\left(\mathbf{u}_{h} \cdot \mathbf{n}\right)\right|_{e_{i}}=\int_{Q} \bar{f}_{h} \phi_{i}-\int_{Q} \mathcal{K} \nabla p_{h} \cdot \nabla \phi_{i} .
$$

Thus, in order to compute the outfluxes through the edges of an element $Q$, we only need to compute the local residual of the solution $p_{h}$ on $Q$. Incidentally, we have proved the existence and uniqueness of a solution of the system (3.2), since $f=0$ implies that $p_{h}=0$ by (3.4), and that $\mathbf{u}_{h}=0$ by (3.5).

Remark 3.1. Our argument applies equally well to triangular grids. In this case, we take $N_{h}$ to be the $P 1$ nonconforming finite element space. In particular, since we have $\nabla N_{h}=\{(1,0),(0,1)\}$, the equations (3.1) and (3.2) are identical. For more details, see [14, [17].

Remark 3.2. Let $h_{x}$ and $h_{y}$ denote the width and height of a rectangle $Q$. Obviously, one can write on $Q$

$$
\mathbf{u}_{h}+\left.\mathcal{K} \nabla p_{h}\right|_{Q}=\left(a+b\left(x-x_{Q}\right), c+d\left(y-y_{Q}\right)\right)
$$

for some constants $a, b, c$ and $d$. In the case of a piecewise constant scalar-valued $\mathcal{K}$, by taking $\nabla \chi=(1,0),(0,1)$ and $(x,-y)$ in (3.2) and using $\operatorname{div}\left(\mathcal{K} \nabla p_{h}\right)=0$, we obtain

$$
a=0, \quad c=0, \quad b h_{x}=d h_{y}, \quad b+d=\bar{f}_{h} .
$$

Hence for a square grid $\left(h_{x}=h_{y}\right)$ and scalar $\mathcal{K}$, one obtains the following formula for $\mathbf{u}_{h}$ :

$$
\left.\mathbf{u}_{h}\right|_{Q}=-\left.\mathcal{K} \nabla p_{h}\right|_{Q}+\frac{\bar{f}_{h}}{2}\left(\mathbf{x}-\mathbf{x}_{Q}\right),
$$

where $\mathbf{x}_{Q}$ is the center of $Q$. The same formula was derived by Chou and Tang [14] in the case of triangular grids.

3.2. FVM on quadrilateral grids. Next we turn to quadrilateral grids. In analogy with the rectangular case, we propose the following scheme:

$$
\left\{\begin{array}{c}
\int_{Q}\left(\mathbf{u}_{h}+\mathcal{K} \nabla p_{h}\right) \cdot \nabla \chi=0, \quad \forall \chi \in N_{h}(Q), \\
\int_{Q} \operatorname{div} \mathbf{u}_{h}=\int_{Q} f .
\end{array}\right.
$$

Elimination of the velocity $\mathbf{u}_{h}$ can be done in the same way as in the rectangular case. Since we again have by (2.11)

$$
\left.\operatorname{div} \mathbf{u}_{h}\right|_{Q}=\frac{1}{J_{Q}} \operatorname{div} \hat{\mathbf{u}}_{h}=\frac{1}{J_{Q}} \int_{Q} f=\left.\bar{f}_{h}\right|_{Q},
$$


consequently (3.3) becomes

$$
\sum_{Q \in \mathcal{Q}_{h}} \int_{Q} \mathbf{u}_{h} \cdot \nabla \chi=-\int_{\Omega} \bar{f}_{h} \chi, \quad \forall \chi \in N_{h},
$$

which gives immediately, by (3.7),

$$
\sum_{Q \in \mathcal{Q}_{h}} \int_{Q} \mathcal{K} \nabla p_{h} \cdot \nabla \chi=\int_{\Omega} \bar{f}_{h} \chi, \quad \forall \chi \in N_{h}
$$

Again this is a rotated- $Q 1$ nonconforming finite element method for the problem (1.1) with a slightly modified right-hand side. Also, the velocity $\mathbf{u}_{h}$ can be recovered from the solution $p_{h}$ by the local residual

$$
\left.\left|e_{i}\right|\left(\mathbf{u}_{h} \cdot \mathbf{n}\right)\right|_{e_{i}}=\int_{Q} \bar{f}_{h} \phi_{i}-\int_{Q} \mathcal{K} \nabla p_{h} \cdot \nabla \phi_{i} .
$$

Remark 3.3. Our techniques can be easily extended to the mixed boundary conditions of general form

$$
\mathbf{u} \cdot \mathbf{n}=g \text { on } \Gamma_{1}, \quad p=h \text { on } \Gamma_{2}, \quad \Gamma_{1} \cup \Gamma_{2}=\partial \Omega, \quad \Gamma_{1} \cap \Gamma_{2}=\emptyset .
$$

\section{ERror estimates}

In this section we derive optimal error estimates for the velocity and the pressure variable. We use the standard notations $|\cdot|_{m, Q}$ and $\|\cdot\|_{m, Q}$ for the semi and full norm of the Sobolev space $H^{m}(Q)$, and set

$$
|\chi|_{1, h}^{2}=\sum_{Q \in \mathcal{Q}_{h}}|\chi|_{1, Q}^{2}, \quad \forall \chi \in H^{1}(\Omega) \oplus N_{h},
$$

and $\|\chi\|_{1, h}=\left(\|\chi\|_{0}^{2}+|\chi|_{1, h}^{2}\right)^{1 / 2}$. We also define the bilinear form

$$
a_{h}(p, \chi)=\sum_{Q \in \mathcal{Q}_{h}} \int_{Q} \mathcal{K} \nabla p \cdot \nabla \chi, \quad \forall p, \chi \in H^{1}(\Omega) \oplus N_{h},
$$

where the subscript $h$ will be dropped when both $p$ and $\chi$ belong to $H^{1}(\Omega)$. To simplify notation, we shall write $|\cdot|_{m},\|\cdot\|_{m}$ instead of $|\cdot|_{m, \Omega},\|\cdot\|_{m, \Omega}$.

Throughout this section we assume elliptic regularity holds. First we prove the following error estimate for the pressure approximation of the finite element method (3.8).

Theorem 4.1. Let $f \in H^{1}(Q), \forall Q \in \mathcal{Q}_{h}$, and let $p_{h} \in N_{h}$ be the solution of (3.8). Then there exists a constant $C$ independent of $h$ such that

$$
\left\|p-p_{h}\right\|_{0}+h\left|p-p_{h}\right|_{1, h} \leq C h^{2}\|f\|_{1, h} .
$$

Proof. Let $\tilde{p} \in H^{2}(\Omega) \cap H_{0}^{1}(\Omega)$ be the solution of

$$
a(\tilde{p}, \chi)=\left(\bar{f}_{h}, \chi\right), \quad \forall \chi \in H_{0}^{1}(\Omega) .
$$

Then we can apply the standard argument of $H^{1}$ estimate and the Aubin-Nitsche technique to obtain (cf. [33])

$$
\left\|\tilde{p}-p_{h}\right\|_{0}+h\left|\tilde{p}-p_{h}\right|_{1, h} \leq C h^{2}\|f\|_{0} .
$$

Now we need to estimate $\|p-\tilde{p}\|_{1}$. Since we have $\int_{Q}\left(f-\bar{f}_{h}\right)=0$, it follows that for all $\chi \in H_{0}^{1}(\Omega)$

$$
a(p-\tilde{p}, \chi)=\left(f-\bar{f}_{h}, \chi\right) \leq C h^{2}\|f\|_{1, h}|\chi|_{1},
$$


where we have used Lemma 2.4. By taking $\chi=p-\tilde{p}$ and using the Poincaré inequality, we obtain

$$
\|p-\tilde{p}\|_{1} \leq C h^{2}\|f\|_{1, h} .
$$

The proof is completed by combining (4.2) and (4.3).

In order to derive the estimate for $\left\|\mathbf{u}-\mathbf{u}_{h}\right\|_{0}$, we note that

$$
\int_{Q}\left[\left(\mathbf{u}-\mathbf{u}_{h}\right)+\mathcal{K} \nabla\left(p-p_{h}\right)\right] \cdot \nabla \chi=0, \quad \forall \chi \in N_{h}(Q),
$$

which, when transferred onto the reference element, becomes

$$
\int_{\hat{Q}}\left[\left(\hat{\mathbf{u}}-\hat{\mathbf{u}}_{h}\right)+J \mathcal{J}^{-1} \mathcal{K} \mathcal{J}^{-t} \hat{\nabla}\left(\hat{p}-\hat{p}_{h}\right)\right] \cdot \hat{\nabla} \hat{\chi}=0, \quad \forall \hat{\chi} \in N_{h}(\hat{Q}) .
$$

Since $\operatorname{div}\left(\hat{\Pi} \hat{\mathbf{u}}-\hat{\mathbf{u}}_{h}\right)=0$, we may take $\hat{\nabla} \hat{\chi}=\hat{\Pi} \hat{\mathbf{u}}-\hat{\mathbf{u}}_{h}$ by Lemma 2.3 to obtain

$$
\begin{aligned}
\left\|\hat{\Pi} \hat{\mathbf{u}}-\hat{\mathbf{u}}_{h}\right\|_{0, \hat{Q}} & \leq\|\hat{\mathbf{u}}-\hat{\Pi} \hat{\mathbf{u}}\|_{0, \hat{Q}}+\|\mathcal{K}\|_{\infty}\|J\|_{\infty, \hat{Q}}\left\|\mathcal{J}^{-1}\right\|_{\infty, Q}^{2}\left|\hat{p}-\hat{p}_{h}\right|_{1, \hat{Q}} \\
& \leq C\left(|\hat{\mathbf{u}}|_{1, \hat{Q}}+\left|\hat{p}-\hat{p}_{h}\right|_{1, \hat{Q}}\right),
\end{aligned}
$$

where we have used the upper bounds (2.4) and (2.5)). Thus it follows by Lemma 2.1 that

$$
\begin{aligned}
\left\|\mathbf{u}-\mathbf{u}_{h}\right\|_{0, Q} & \leq\left\|\hat{\mathbf{u}}-\hat{\mathbf{u}}_{h}\right\|_{0, \hat{Q}} \leq\|\hat{\mathbf{u}}-\hat{\Pi} \hat{\mathbf{u}}\|_{0, \hat{Q}}+\left\|\hat{\Pi} \hat{\mathbf{u}}-\hat{\mathbf{u}}_{h}\right\|_{0, \hat{Q}} \\
& \leq C\left(|\hat{\mathbf{u}}|_{1, \hat{Q}}+\left|\hat{p}-\hat{p}_{h}\right|_{1, \hat{Q}}\right) \\
& \leq C\left(h|\mathbf{u}|_{1, Q}+\left|p-p_{h}\right|_{1, Q}\right),
\end{aligned}
$$

which gives by Theorem 4.1

$$
\left\|\mathbf{u}-\mathbf{u}_{h}\right\|_{0} \leq C h\left(\|\mathbf{u}\|_{1}+\|f\|_{1, h}\right)
$$

Finally, the estimate for $\left\|\operatorname{div} \mathbf{u}-\operatorname{div} \mathbf{u}_{h}\right\|_{0}$ follows directly from Lemma 2.4. since we have $\operatorname{div} \mathbf{u}=f$ and $\operatorname{div} \mathbf{u}_{h}=\bar{f}_{h}$. We summarize these results in the following theorem.

Theorem 4.2. Let $\left(\mathbf{u}_{h}, p_{h}\right)$ be the solution of the system [3.7. Then there exists a constant $C$ independent of $h$ such that

$$
\left\|\mathbf{u}-\mathbf{u}_{h}\right\|_{0}+\left\|\operatorname{div} \mathbf{u}-\operatorname{div} \mathbf{u}_{h}\right\|_{0} \leq C h\left(\|\mathbf{u}\|_{1}+\|f\|_{1, h}\right),
$$

provided that $p$ is in $H^{2}(\Omega)$, and the $L^{2}$ function $f$ is locally in $H^{1}(Q), \forall Q \in \mathcal{Q}_{h}$.

\section{Numerical RESUlts}

To confirm the theoretical results established in the previous section, some numerical tests are carried out on the unit square $\Omega=(0,1)^{2}$, involving scalar and tensor coefficients, smooth and nonsmooth coefficients, and rectangular and quadrilateral grids. For convenience, we compute velocity error in an $L^{2}$ seminorm and pressure error in a discrete $L^{2}$ norm:

$$
\begin{gathered}
\delta_{u}=\left\{\sum_{Q \in \mathcal{Q}_{h}} \sum_{e \in \partial Q}\left[\int_{e}\left(\mathbf{u}-\mathbf{u}_{h}\right) \cdot \mathbf{n} d s\right]^{2}\right\}^{1 / 2}, \\
\delta_{p}=\left[\sum_{Q \in \mathcal{Q}_{h}}|Q|\left(p\left(\mathbf{x}_{Q}\right)-p_{h}\left(\mathbf{x}_{Q}\right)\right)^{2}\right]^{1 / 2}
\end{gathered}
$$


where the edge integrals are evaluated by the midpoint rule, and $\mathbf{x}_{Q}$ is the mass center of $Q$. These discrete (semi)norms are the ones defined in [14 16], except that $\delta_{u}$ is defined elementwise and thus involves the interior edge integrals twice.

In all of the examples below, the exact solutions $p$ and the coefficients $\mathcal{K}$ are given in explicit form, and the source terms $f$ and the boundary conditions are determined by them. In the first three examples we partition $\Omega$ into squares of size $h$, whereas we consider a distorted grid in the last example (cf. Figure 5.1).

Based on the computed errors for the problems, we also report the convergence rates in Table 5, assuming that the error is of the form $C h^{\alpha}$. Here $C$ and $\alpha$ are determined by the least squares fit to the data. One notes the superconvergence behavior of the flux at the midpoints.

Problem 1. $\mathcal{K}=1.0$ and $p(x, y)=x(1-x) \sin (\pi y)$

Problem 2. $\mathcal{K}=1+10 x+y$ and $p(x, y)=x(1-x) y(1-y)$

Problem 3. This example is the one presented in [25]:

$$
\mathcal{K}=\left(\begin{array}{cc}
\frac{14}{9} & \frac{7}{9} \\
\frac{7}{9} & 2
\end{array}\right) \text { for } 0<x<.5, \quad\left(\begin{array}{cc}
1 & \frac{1}{2} \\
\frac{1}{2} & 2
\end{array}\right) \text { for } .5<x<1
$$

and

$$
p(x, y)= \begin{cases}1-x^{3} & \text { for } 0<x<.5 \\ \frac{7}{6}\left(1-x^{2}\right) & \text { for } .5<x<1\end{cases}
$$

By simple calculations it is easy to see that the velocity $\mathbf{u}=-\mathcal{K} \nabla p$ has continuous normal components across the line of discontinuity $x=1 / 2$.

Problem 4. This example is the one presented in [6, 25]:

$$
\mathcal{K}=\left(\begin{array}{cc}
\cos \theta & \sin \theta \\
-\sin \theta & \cos \theta
\end{array}\right)\left(\begin{array}{cc}
1 & 0 \\
0 & 0.01
\end{array}\right)\left(\begin{array}{cc}
\cos \theta & -\sin \theta \\
\sin \theta & \cos \theta
\end{array}\right),
$$

and $p(x, y)=\cos (\pi x) \cos (2 \pi y)$. The geometry of the grid is shown in Figure 5.1 .
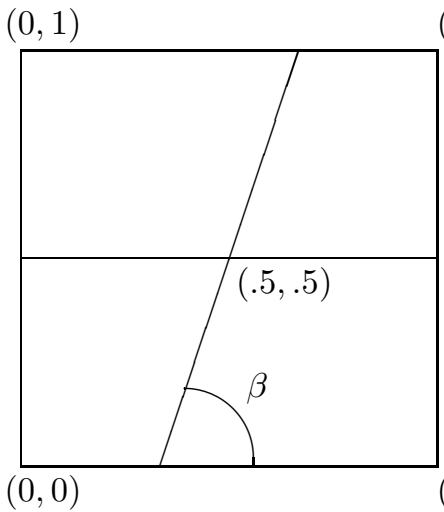

$(1,0)$

Figure 5.1. Distorted grids for Problem 4 
Table 1. Problem 1: Constant scalar coefficients

\begin{tabular}{|c|c|c|}
\hline$h$ & $\delta_{u}$ & $\delta_{p}$ \\
\hline $1 / 8$ & $5.9935 \mathrm{e}-3$ & $3.0080 \mathrm{e}-3$ \\
\hline $1 / 16$ & $1.4992 \mathrm{e}-3$ & $7.5270 \mathrm{e}-4$ \\
\hline $1 / 32$ & $3.7483 \mathrm{e}-4$ & $1.8822 \mathrm{e}-4$ \\
\hline $1 / 64$ & $9.3711 \mathrm{e}-5$ & $4.7058 \mathrm{e}-5$ \\
\hline $1 / 128$ & $2.3428 \mathrm{e}-5$ & $1.1765 \mathrm{e}-5$ \\
\hline
\end{tabular}

TABLE 2. Problem 2: Variable scalar coefficients

\begin{tabular}{|c|c|c|}
\hline$h$ & $\delta_{u}$ & $\delta_{p}$ \\
\hline $1 / 8$ & $2.0213 \mathrm{e}-2$ & $6.9621 \mathrm{e}-4$ \\
\hline $1 / 16$ & $5.0450 \mathrm{e}-3$ & $1.7362 \mathrm{e}-4$ \\
\hline $1 / 32$ & $1.2608 \mathrm{e}-3$ & $4.3377 \mathrm{e}-5$ \\
\hline $1 / 64$ & $3.1515 \mathrm{e}-4$ & $1.0843 \mathrm{e}-5$ \\
\hline $1 / 128$ & $7.8784 \mathrm{e}-5$ & $2.7105 \mathrm{e}-6$ \\
\hline
\end{tabular}

TABLE 3. Problem 3: Discontinuous tensor coefficients

\begin{tabular}{|c|c|c|}
\hline$h$ & $\delta_{u}$ & $\delta_{p}$ \\
\hline $1 / 8$ & $1.4378 \mathrm{e}-2$ & $3.0216 \mathrm{e}-3$ \\
\hline $1 / 16$ & $3.6223 \mathrm{e}-3$ & $7.5599 \mathrm{e}-4$ \\
\hline $1 / 32$ & $9.1484 \mathrm{e}-4$ & $1.8904 \mathrm{e}-4$ \\
\hline $1 / 64$ & $2.3118 \mathrm{e}-4$ & $4.7262 \mathrm{e}-5$ \\
\hline $1 / 128$ & $5.8414 \mathrm{e}-5$ & $1.1816 \mathrm{e}-5$ \\
\hline
\end{tabular}

TABLE 4. Problem 4: Distorted grids, $\beta=60^{\circ}, \theta=45^{\circ}$

\begin{tabular}{|c|c|c|}
\hline Grid Size & $\delta_{u}$ & $\delta_{p}$ \\
\hline $8 \times 8$ & $2.0878 \mathrm{e}-1$ & $4.1977 \mathrm{e}-2$ \\
\hline $16 \times 16$ & $5.2684 \mathrm{e}-2$ & $1.0989 \mathrm{e}-2$ \\
\hline $32 \times 32$ & $1.3526 \mathrm{e}-2$ & $2.7816 \mathrm{e}-3$ \\
\hline $64 \times 64$ & $3.4843 \mathrm{e}-3$ & $6.9757 \mathrm{e}-4$ \\
\hline $128 \times 128$ & $8.9701 \mathrm{e}-4$ & $1.7453 \mathrm{e}-4$ \\
\hline
\end{tabular}

TABLE 5. Convergence rates: $\delta_{u} \leq C_{u} h^{\alpha_{u}}$ and $\delta_{p} \leq C_{p} h^{\alpha_{p}}$

\begin{tabular}{|c|c|c|c|c|}
\hline & $C_{u}$ & $\alpha_{u}$ & $C_{p}$ & $\alpha_{p}$ \\
\hline Problem 1. & 0.384 & 1.999 & 0.193 & 1.999 \\
\hline Problem 2. & 1.295 & 2.000 & 0.045 & 2.001 \\
\hline Problem 3. & 0.893 & 1.985 & 0.194 & 1.999 \\
\hline Problem 4. & 12.308 & 1.964 & 2.622 & 1.979 \\
\hline
\end{tabular}




\section{REFERENCES}

1. D. N. Arnold And F. BrezzI, Mixed and nonconforming finite element methods : implementation, postprocessing and error estimates, RAIRO Model. Math. Anal. Numer. 19 (1985), pp. 7-32. MR 87g:65126

2. S. C. Brenner, An optimal order multigrid for P1 nonconforming finite elements, Math. Comp. 52 (1989), pp. 1-15. MR 89f:65119

3. F. Brezzi, J. Douglas, M. Fortin and L. Marini, Efficient rectangular mixed finite elements in two and three variables, RAIRO Model. Math. Numer. Anal. 21 (1987), pp. 581-604. MR 88j:65249

4. F. BRezzi, J. Douglas, And L. Marini, Two families of mixed finite elements for second order elliptic problems, Numer. Math. 47 (1985), pp. 217-235. MR 87g:65133

5. F. Brezzi And M. Fortin, Mixed and hybrid finite element methods, Springer-Verlag, NewYork, (1991). MR 92d:65187

6. Z. Cai, J. E. Jones, S. F. McCormick and T. F. Russell, Control-volume mixed finite element Methods, Computational Geosciences 1 (1997), pp. 289-315. MR 2000a:76106

7. Z. CHEN, Analysis of mixed methods using conforming and nonconforming finite element methods, RAIRO Model. Math. Anal. Numer. 27 (1993), pp. 9-34. MR 94c:65132

8. Z. CHEN, Multigrid algorithms for mixed methods for second order elliptic problems, IMA Preprint Series \#1218, March 1994.

9. Z. Chen AND P. Oswald, Multigrid and multilevel methods for nonconforming Q1 elements, Math. Comp. 67 (1998), pp. 667-693. MR 98g:65118

10. S. H. ChOu And S. He, On the regularity and uniformness conditions on quadrilateral grids, submitted to Comput. Methods Appl. Mech. Engrg., (2001).

11. S. H. ChOU AND D. Y. KWAK, Mixed covolume methods on rectangular grids for elliptic problems, SIAM J. Numer. Anal. 37, No. 3 (2000). pp. 758-771. MR 2001c:65143

12. S. H. Chou, D. Y. KWAK And P. VASsilevski, Mixed covolume methods for elliptic problems on triangular grids, SIAM J. Numer. Anal. 35, No. 5 (1998). pp. 1850-1861. MR 99k:65105

13. S. H. ChOU, D. Y. KWAK AND K. Y. KIM, A general framework for constructing and analyzing mixed finite volume methods on quadrilateral grids: the overlapping covolume case, accepted for publication in SIAM J. Numer. Anal. (2001).

14. S. H. CHOU AND S. TANG, Conservative P1 conforming and nonconforming Galerkin FEMs: effective flux evaluation via a nonmixed method approach, SIAM J. Numer. Anal. 38 (2000). pp. 660-680. CMP 2000:16

15. S. H. ChOU AND S. TANG, Comparing two approaches of analyzing mixed finite volume methods, BGSU report, Bowling Green State University, OH. (2000).

16. S. H. Chou And P. S. VAssilevski, A general mixed covolume framework for constructing conservative schemes for elliptic problems, Math. Comp., 68, No. 227, (1999). pp. 991-1011. MR 99j:65203

17. B. Courbet And J. P. Croisille, Finite volume box schemes on triangular meshes, RAIRO Model. Math. Anal. Numer. 32 (1998), pp. 631-649. MR 99i:65124

18. J. Douglas, JR., R. E. Ewing And M. F. Wheeler, The approximation of the pressure by a mixed method in the simulation of miscible displacement, RAIRO Anal. Numér. 17 (1983), pp. 17-33.

19. J. Douglas, JR. And J. E. Roberts, Global estimates for mixed methods for second order elliptic equations, Math. Comp. 44 (1985), pp. 39-52. MR 84f:76047

20. R. FAlK And J. Osborn, Error estimates for mixed methods, RAIRO Anal. Numér. 14 (1980), pp. 249-277. MR 82j:65076

21. M. FORTIN, An analysis of the convergence of mixed finite element methods, RAIRO Anal. Numér. 11 (1977), pp. 341-354. MR 57:4473

22. Fraeijis de Veubeke B., Displacement and equilibrium models in the finite element method, in Stress Analysis (O.C. Zienkiewicz and G. Holister, eds), John Wiley and Sons, New York(1965).

23. V. GiRAult And P. A. RAVIART, Finite element methods for Navier-Stokes equations, Theory and Algorithms, Springer-Verlag, Berlin, 1986. MR 88b:65129

24. C. Johnson And V. Thomee, Error estimates for some mixed finite element methods for parabolic type problems, RAIRO Anal. Numér 15 (1981), pp. 41-78. MR 83c:65239

25. J. E. JONEs A mixed finite volume element method for accurate computation of fluid velocities in porous media, $\mathrm{Ph}$. D. thesis, University of Colorado at Denver, (1995). 
26. C. LEE, A nonconforming multigrid method using conforming subspaces, In the Proceedings of the Sixth Copper Mountain Conference on Multigrid Methods, Eds: N. D. Melson and T. A. Manteuffel and S. F. McCormick, (1993), pp 317-330.

27. L. D. MARINI, An inexpensive method for the evaluation of the solution of the lowest order Raviart-Thomas mixed method, SIAM J. Numer. Anal. 22 (1995), pp. 493-496. MR 86g:65214

28. L. D. MARIni And P. Pietra, An abstract theory for mixed approximations of second order elliptic problems, Mat. Aplic. Comp. 8 (1989), pp. 219-239. MR 91h:65177

29. S. McCormick, Multilevel adaptive methods for partial differential equations, Society of Industrial and Applied Mathematics, Philadelphia, PA, (1989). MR 91h:65206

30. M. OHLBerger, Convergence of a mixed finite elements-finite volume method for the two phase flow in porous media, East-West J. Numer. Math. 5 (1997), pp.183-210. MR 98m:76105

31. K. Pruess, TOUGH user's guide, Nuclear Reulatory Commission, report NUREG/CR-4645, (1987).

32. K. Pruess, TOUGH2: A general numerical simulator for multiphase fluid and heat flow, LBL report LBL-29400, Berkeley, California, (1991).

33. R. Rannacher and S. Turek, Simple nonconforming quadrilateral Stokes element, Numer. Methods in Partial Diff. Eqns. 8 (1992), pp. 97-111. MR 92i:65170

34. P. A. Raviart And J. M. Thomas, A mixed finite element method for 2 nd order elliptic problems, in Proc. Conf. on Mathematical Aspects of Finite Element Methods, Lecture Notes in Math., Vol. 606, Springer-Verlag, Berlin, 1977, pp. 292-315. MR 58:3547

35. R. SACCO AND F. SAlERI, Stabilization of mixed finite elements for convection-diffusion problems, CWI Quarterly, 10 (1997), pp. 301-315. MR 99d:65326

36. R. SACCO AND F. SALERI, Mixed ffinite volume methods for semiconductor device simulation, Numer. Methods in Partial Diff. Eqns. 13 (1997), pp. 215-236. MR 98a:65181

37. E. Suli, The accuracy of cell vertex finite volume methods on quadrilateral meshes, Math. Comp, 59 (1992), pp. 359-382. MR 93a:65158

38. J.-M. Thomas And D. Trujillo, Mixed finite volume methods, Inter. J. Numer. Methods Eng. 46 (1999), pp. 1351-1366. MR 2000i:65158

39. J. Wang And T. Mathew, Mixed finite element methods over quadrilaterals, In the Proceedings of the Third International Conference on Advances in Numerical Methods and Applications, Eds: I. T. Dimov, Bl. Sendov, and P. Vassilevski, World Scientific, (1994), pp $203-214$.

Department of Mathematics and Statistics, Bowling Green State University, BowlING Green, Ohio 43403

E-mail address: chou@bgnet.bgsu.edu

Department of Mathematics, Korea Advanced Institute of Science and Technology, TAEJON, KOREA 305-701

E-mail address: dykwak@math.kaist.ac.kr

Department of Mathematics, Korea Advanced Institute of Science and Technology, TAEJON, Korea 305-701

E-mail address: kky@mathx.kaist.ac.kr 